\title{
A hypomorphic allele of telomerase reverse transcriptase uncovers the minimal functional length of telomeres in Arabidopsis.
}

J. Matthew Watson ${ }^{1}$, Johanna Trieb ${ }^{1}$, Martina Troestl ${ }^{1}$, Kyle Renfrew ${ }^{2,4}$, Terezie Mandakova ${ }^{3}$, Dorothy E. Shippen ${ }^{4}$, Karel Riha ${ }^{3 *}$

${ }^{1}$ Gregor Mendel Institute of Plant Molecular Biology, Dr. Bohr-Gasse 3, 1030 Vienna, Austria

${ }^{2}$ Current affiliation: Thermo Fisher Scientific, 2215 Grand Avenue Parkway, Austin, TX 78728, USA

${ }^{3}$ Central European Institute of Technology, Masaryk University, Kamenice 5 , Brno, Czech Republic

${ }^{4}$ Texas A\&M University, Dept. of Biochemistry, 2128 TAMU, College Station TX 77840, USA

${ }^{*}$ Corresponding author. karel.riha@ceitec.muni.cz

Keywords: Telomeres, telomerase, genome stability, subtelomere, t-loop 


\begin{abstract}
Despite the essential requirement of telomeric DNA for genome stability, the length of telomere tracts between species differs by up to four orders of magnitude, raising the question of the minimal length of telomeric DNA necessary for proper function. Here we address this question using a hypomorphic allele of the telomerase catalytic subunit, TERT. We show that although this construct partially restored telomerase activity to a tert mutant, telomeres continued to shorten over several generations, ultimately stabilizing at a bimodal size distribution. Telomeres on two chromosome arms were maintained at a length of $1 \mathrm{~kb}$, while the remaining telomeres were maintained at $400 \mathrm{bp}$. The longest telomeres identified in this background were also significantly longer in wild type populations, suggesting cis-acting elements on these arms either promote telomerase processivity or recruitment. Genetically disrupting telomerase processivity in this background resulted in immediate lethality. Thus, telomeres of $400 \mathrm{bp}$ are both necessary and sufficient for Arabidopsis viability. As this length is the estimated minimal length for t-loop formation, our data suggest that telomeres long enough to form a t-loop constitute the minimal functional length.
\end{abstract}




\section{Introduction}

The ends of most eukaryotic chromosomes are capped by telomeres that serve two primary functions: they distinguish the natural chromosome end from a DNA double-strand break (DSB) and provide a mechanism for overcoming the end-replication problem (Fouquerel, Parikh et al., 2016, Stewart, Chaiken et al., 2012). Telomeres are composed primarily of repetitive telomeric DNA and telomere specific DNA binding proteins (de Lange, 2010). The telomeric repeat is a short G-rich sequence, TTAGGG in vertebrates and TTTAGGG in plants (Fulcher, Derboven et al., 2014). Telomeric DNA cannot be fully replicated by conventional DNA polymerases, an issue known as the endreplication problem. During lagging strand synthesis, removal of the final RNA primer results in the formation of a gap in the DNA that cannot be replaced, leading to the formation of a 3 ' single-stranded DNA overhang (G-overhang) and a small loss of terminal sequences (Soudet, Jolivet et al., 2014). The end-replication problem can be counteracted by the enzyme telomerase, consisting minimally of the catalytic subunit TERT (telomerase reverse-transcriptase) and an internal RNA template, TER (telomerase RNA) (Greider \& Blackburn, 1989). Telomerase can anneal to the G-overhang and, using its internal template, processively extend the extreme $3^{\prime}$ end (Tomita, 2018).

Telomeric repeats serve as a platform for sequence-specific telomere binding proteins that can associate either with double- or single-stranded DNA. In mammals, double-strand telomere binding proteins TRF1 and TRF2 anchor a multi-subunit protein complex termed shelterin to telomeres (de Lange, 2010). Shelterin is necessary for preventing cells from recognizing telomeres as DSBs (Sfeir \& de Lange, 2012), but precisely how this is accomplished is unknown. One model predicts that chromosome ends are protected through the formation of t-loops. T-loops are higher order structures in which the G-overhang invades duplex telomeric DNA, forming a looped molecule that hides the chromosome 3' terminal extension (Doksani, Wu et al., 2013, Griffith, Comeau et al., 1999). Although first described in mammals, T-loops have also been identified in other taxa including plants (Cesare, Quinney et al., 2003, Munoz-Jordan, Cross et al., 2001, Raices, Verdun et al., 2008, Tomaska, Makhov et al., 2002). T-loops likely do not form in organisms that possess relatively short telomeres, such as Saccharomyces cerevisiae or in the vegetative nuclei of ciliates (Luke-Glaser, Poschke et al., 2012, Tomaska, Willcox et al., 2004). In these species, chromosome end protection appears to largely depend on protein complexes associated with single-stranded telomeric DNA (Greetham, Skordalakes et al., 2015).

In the absence of telomerase, telomeric DNA gradually shortens, eventually leading to telomere dysfunction. Thus, the ability of telomeres to protect chromosome ends and allow for cellular viability is a function of their length. The molecular details of this relationship are unclear. Telomeres are maintained at different lengths in different organisms, ranging from 300 bp in yeasts to as long as $150 \mathrm{~kb}$ in tobacco and the telomere length can also be highly variable within a species (Fajkus, Kovarik et al., 1995, Fulcher, Teubenbacher et al., 2015, Liti, Haricharan et al., 2009, Shakirov \& Shippen, 2004). Most organisms keep telomeres at lengths longer than necessary for function to provide a protective buffer, as defects in telomere maintenance are often only apparent after multiple generations (Blasco, Lee et al., 1997, Lundblad \& Szostak, 1989, Meier, Clejan et al., 2006, Nakamura, Morin et al., 1997, Riha, McKnight et al., 2001). Several attempts have been made to determine the minimal length of telomeric DNA necessary for telomere function (Capper, Britt-Compton et al., 2007, Heacock, Spangler et al., 2004, Xu \& Blackburn, 2007). These approaches have relied on the identification of the smallest telomere within a population undergoing telomere crisis due to ongoing telomere shortening. The minimal functional lengths inferred from these experiments vary from 
roughly 100 bp in yeast (Forstemann, Hoss et al., 2000, Teixeira, Arneric et al., 2004), 42-78 bp in human cell lines (Capper et al., 2007, Xu \& Blackburn, 2007), and 360 bp in Arabidopsis (Heacock et al., 2004). However, it has not been possible to determine whether the very short telomeres identified in these studies are actually functional, since they form only a small portion of total telomeres and may represent dysfunctional and nucleolytically processed intermediates. Because variations in minimal telomere length may belie fundamental differences in telomere capping, determining the amount of telomeric DNA necessary for telomere function can provide insight into mechanisms governing chromosome-end protection.

Here we report the creation of Arabidopsis strains that maintain their telomeres at the minimal functional threshold by expressing a hypomorphic allele of TERT. While plants with these shortened telomeres exhibit chromosome end-to-end fusions and ongoing genome instability, they are capable of indefinite survival as long as they are not subjected to further telomere erosion. Thus, we propose that the final telomere length reached in this population, roughly $400 \mathrm{bp}$, represents the minimal length necessary for proper telomere function in Arabidopsis.

\section{Results}

Following the discovery and analysis of telomerase mutants in Arabidopsis, we attempted to study the fate of shortened telomeres upon reintroduction of telomerase activity. To this end, we transformed $4^{\text {th }}$ generation tert mutants (G4) with constructs expressing the TERT CDNA under the control of either the cauliflower mosaic virus $35 \mathrm{~S}$ or actin (ACT2) promoter (Fig. S1). While both constructs restored telomerase activity, TRAP assays revealed that enzyme activity was significantly reduced compared to wild type plants. Plants bearing the ACT2:TERT construct showed slightly higher levels of telomerase than the 35S:TERT lines (Fig. 1A).

We followed two independent transformant lines for both constructs through multiple generations (Fig. S1) and measured telomere length by terminal restriction fragment length analysis (TRF) in G7/T3 and G8/T4. Telomeres in the two lines expressing TERT driven by the actin promoter showed a gradual lengthening of telomeres (Fig. 1B), consistent with restoration of telomerase function in telomere synthesis. In contrast, both lines in which TERT expression was driven by the $35 \mathrm{~S}$ promoter exhibited continual shortening of telomeres. The telomeric signal was under $1.5 \mathrm{~kb}$ in G7/T3 in 35S:TERT plants and telomeres further shortened in G8/T4 (Fig. 1B). Telomeres were also substantially shorter than telomeres of parental G5 tert plants. Additionally, telomeres in the 35S:TERT lines displayed a discrete TRF banding pattern, a feature typical for plants with inactive telomerase. These data indicate that despite detectable enzyme activity, telomerase in the 35S:TERT lines is incapable of counteracting the end-replication problem and telomeres continue to shorten.

To follow the fate of lines expressing the hypomorphic TERT allele, we continued to propagate the two 35S:TERT lines, named 2 and 7, for multiple generations. Unexpectedly, despite the progressive loss of telomeric DNA observed in the initial TRFs (Fig. 1), we were able to self-propagate both line 2 and line 7 through generation G18/T14, when the experiment was discontinued. This is ten generations longer than tert null mutants, which arrest growth in a terminal generation of G8 - G10 (Riha et al., 2001). Notably, out-segregation of the 35S:TERT construct in G7/T3 resulted in termination of the population in the next generation (Fig. S1), confirming that the 35S:TERT construct was responsible for survival of complemented tert mutants. Despite the ability of the 35S:TERT lines to 
survive for multiple generations, they began to display phenotypes characteristic of genomic instability within two generations after transformation (G7/T3, Fig. 1). These phenotypes including wrinkled leaves, fasciated stems, altered phyllotaxy, and reduced seed set. Plants with these phenotypes have previously been categorized based on increasing severity into Type I, Type II, and Terminal phenotypes (Riha et al., 2001), with Type I plants showing mild defects in leaf morphology and phyllotaxy, Type II plants exhibiting severe developmental defects and reduced fertility, and dwarfed terminal plants that do not produce any seeds. We scored populations of plants from both 35S:TERT lines for the frequency of these phenotypic categories over multiple generations (Fig. 1C). The severity of the phenotypes increased with each successive generation until G12/T8, indicative of increasing telomere dysfunction. In accordance with the increasing severity of the phenotypes, we observed a gradual increase in the frequency of anaphase bridges through G12/T8 (Fig. 1D), suggesting that the worsening phenotypes are due to increased genomic instability caused by telomere erosion. Notably, the fraction of plants within the respective phenotypic categories became stabilized in subsequent generations after G12/T8, with approximately one third of plants exhibiting wild type or Type I phenotypes (Fig. 1C and Fig. S2). This observation suggests that surviving populations exhibit ongoing genome instability and partial telomere dysfunction.

Several reports have indicated that telomerase has biological functions beyond its primary role of extending telomeres (Blasco, 2002, Li \& Tergaonkar, 2014). To test whether the extended lifespan of the 35S:TERT lines is due to a non-canonical function of telomerase, we generated a TERT CDNA construct containing a point mutation (D860N) in a conserved catalytic residue necessary for telomerase function (Nakayama, Tahara et al., 1998). This construct was transformed into G4 tert plants, and two independent transformants were analyzed for telomerase activity and long-term survival. As expected, we were unable to detect telomerase activity from the 35S:TERT(D860N) lines (Fig. S3). The plants carrying the catalytically dead TERT construct survived for only two generations after transformation, essentially phenocopying the parental tert mutants (Fig S1, Fig. 2A). We conclude that the continued survival of 35S:TERT plants is due to telomerase activity, and not due to a noncanonical function of the TERT protein.

To determine whether the 35S:TERT construct acts through the canonical telomere maintenance pathway, we examined its genetic interaction with other factors known to be involved in regulating telomerase activity at telomeres in Arabidopsis. We first asked whether the 35S:TERT allele could extend telomeres in the absence of KU70, a negative regulator of telomerase whose inactivation results in telomerase-dependent telomere lengthening (Riha \& Shippen, 2003, Riha, Watson et al., 2002). We generated ku70 tert 35:TERT plants by crossing (Fig. 2A and S4). Loss of KU70 in the 35S:TERT background led to an immediate and obvious lengthening of telomeres, demonstrating that despite its reduced activity (Fig. 1A), the 35S:TERT telomerase is capable of extending telomeres. Next, we asked whether survival of the 35S:TERT lines depends on POT1A, an OB-fold containing protein that directly interacts with the telomerase RNP and is required for telomerase repeat addition processivity (Renfrew, Song et al., 2014, Surovtseva, Shakirov et al., 2007). Plants deficient for POT1A exhibit an ever-shorter telomere phenotype, and this phenotype is epistatic with TERT (Surovtseva et al., 2007). We crossed third generation tert pot1a double mutants to T2 35S:TERT plants from line 2 (Fig. S5), and propagated the progeny for an additional three generations as $P O T 1 A^{+/}$, allowing the longer telomeres originating from the tert pot1a parent to shorten. We then outsegregated pot1a mutants in $\mathrm{F} 5$ and F6 and scored the population for growth phenotypes (Fig. $\mathrm{S} 5$ and $2 \mathrm{C}$ ). Population of POT1 $A^{-/}$mutants in both generations showed more severe phenotypes than either the POT1 $A^{+/+}$or POT1 $A^{+/}$plants, and all 
pot $1 a^{-/}$plants in the $\mathrm{F} 6$ population displayed terminal phenotype. These results demonstrate that the survival of 35S:TERT lines requires POT1A.

Taken together, these genetic experiments suggest that the long-term survival of the 35S:TERT lines depends on the ability of telomerase to add telomeric repeats to the telomeres. We hypothesized that the 35S:TERT construct leads to the formation of a hypomorphic telomerase enzyme that is unable to rescue the shortening of telomeres in tert mutants, but supports continuous growth in a significant fraction of the population by maintaining telomeres once they reach minimal length necessary for telomere protection. To test this prediction, we analyzed telomeres in 35S:TERT lines by PETRA, a PCR based method that permits precise analysis of telomere length at individual chromosome arms (Heacock et al., 2004). We measured the length of telomeres at seven chromosome arms in four to five sibling plants from each line, every two generations, between G6 /T2 and G14/T10 (Fig. 3A). The plants selected for analysis were randomly sampled from the pool of plants displaying Type I and Type II phenotypes. At the beginning of the experiment in G6/T2, telomeres ranged in size from a low of $285 \mathrm{bp}$ to a high of $1797 \mathrm{bp}$ with an average length of $951 \pm 350 \mathrm{bp}$. In line 2, telomeres that began with a length above $1 \mathrm{~kb}$ shortened at an average rate of $243 \pm 102 \mathrm{bp}$ per generation, and this rate continued through G10/T6 in the longest telomeres (1R, 2R, and $5 \mathrm{~L})$. We observed a similar trend in line 7 , where telomeres longer than $1 \mathrm{~kb}(1 \mathrm{~L}, 2 \mathrm{R}$, and $5 \mathrm{~L})$ shortened with an average rate of $198 \pm 80$ bp per generation. A similar rate of telomere shortening has been reported for tert mutants (Riha et al., 2001, Watson \& Shippen, 2007), suggesting that the hypomorphic telomerase is not extending these telomeres. In contrast to the longest telomeres, the shortest telomeres in both lines remained essentially unchanged throughout the eight generations of the experiment (Fig. 3A, $1 \mathrm{~L}$ and $5 \mathrm{R}$ in line $2,5 R$ in line 7$)$, indicating that they are sustained at these lengths by the hypomorphic telomerase. Additionally, while the longest telomeres shortened quite rapidly between G6/T2 and G10/T6, the rate of shortening became negligible from G10/T6 onwards, and telomere length was stable for the remaining four generations of the experiment.

While the distribution of starting telomere lengths in the two lines varied from 300 to $1800 \mathrm{bp}$, the final distribution was strongly bimodal, with most telomeres clustered around 400 bp (Fig. 3B 1L, $1 R, 3 L, 5 L, 5 R ; 421 \pm 121$ bp), and two telomeres clustered around 900 bp (Fig. 3B 2R, 4R; $910 \pm 115$ bp). Interestingly, despite varying starting lengths between the two lines, each individual telomere shortened to a similar final length in both lines. As apparent from Fig. 3A, the length of each telomere at G14/T10, was already reached in most cases by G10/T6, and for the shortest telomeres even from the beginning of the experiment in $\mathrm{G} 6 / \mathrm{T} 2$. Although we terminated the experiment at generation G18/T14, the relative stability of telomeres from G10/T6 onwards implies that the hypomorphic telomerase was capable of maintaining telomeres at this length indefinitely. Our results further suggest that the hypomorphic telomerase is capable of maintaining telomeres only when they reach a chromosome-arm specific length. Since these late generation 35S:TERT lines exhibit ongoing chromosome end-to-end fusions (Fig. 1D), it is likely that the ability of telomerase to extend particular telomeres directly correlates with their capping status; fully functional telomeres are not extendable, but telomeres that become partially deprotected are substrates for the hypomorphic telomerase. Along with the observation that further disruption of the telomerase pathway leads to lethality, we conclude that the 35S:TERT lines maintain telomeres close to their minimal functional length.

The bimodal size distribution of telomere lengths in G14/T10 was intriguing, as it suggested that the length at which chromosome ends become accessible to the hypomorphic telomerase is telomere-specific. Therefore, we asked whether the two longer telomeres, $2 \mathrm{R}$ and $4 \mathrm{R}$, were more 
stably capped than the others. To test this, we performed pairwise comparison of the frequency of anaphase bridges involving short and long telomeres by FISH in G14/T10 35S:TERT lines (Fig. 3C). As expected based on the phenotypic defects as well as the large numbers of anaphase bridges detected in late generation 35:TERT plants, telomere to telomere fusions were readily detectable. Despite the large difference in telomere length, $2 R$ and $4 R$ were equally as likely to be involved in chromosome fusions as the shorter arms $1 R$ and $1 \mathrm{~L}$ (Table 1 ). This indicates that $2 \mathrm{R}$ and $4 \mathrm{R}$ telomeres become deprotected at longer lengths relative to the other chromosome arms.

The dramatic difference in telomere length of $2 R$ and $4 R$ suggests that cis acting elements on these two subtelomeric arms regulate telomere homeostasis differently than on the other chromosome arms we have tested. We therefore examined telomere length in wild type plants to determine whether these two telomeres are differentially regulated under normal conditions as well. To accomplish this, we measured telomere length by STELA in 32 wild type plants from four bulk seed stocks. While in PETRA assay are the PCR adaptors anchored to the chromosome end via primer extension through telomeres, ligation is used in STELA (Capper et al., 2007, Heacock et al., 2004). We chose STELA over PETRA in this analysis due to concern that the primer extension reaction may not proceed through the entire length of wild type telomeres. Telomere length was measured as the length of the peak signal, and for allelic telomeres the length of the two alleles was averaged. As shown in Fig. 4A, the $2 \mathrm{R}$ and $4 \mathrm{R}$ telomeres were consistently longer than other telomeres in wild type plants as judged by two alternate methods. These data argue that cis acting elements affect the length of telomere homeostasis in the hypomorphic mutant as well as in wild type.

\section{Discussion}

In this study we describe a hypomorphic allele of TERT that produces a partially functional telomerase. Although this enzyme is incapable of maintaining telomeres within the wild type size range, it nevertheless sustains telomeres when they shorten to a length at which they become fusogenic. The enzyme also retains the capability to extend telomeres in plants depleted of Ku70. The $\mathrm{Ku}$ complex inhibits telomerase in Arabidopsis, and it also protects telomeres by preventing their exonucleolytic degradation and homologous recombination (Kazda, Zellinger et al., 2012, Riha \& Shippen, 2003). Together, our data indicate that telomerase derived from the 35S:TERT allele only accesses telomeres that are not fully capped. The molecular mechanism underlying the impaired telomerase performance in the 35S:TERT lines is not known. It may be due to a reduced level of telomerase that is apparent from in vitro activity assay in comparison with wild type and ACT2:TERT plants. Notably, while the 35S:TERT construct lacks introns, the ACT2:TERT construct contains an intron in the $5^{\prime}$ UTR. It is well established that presence of introns enhances transgene expression in plants (Laxa, 2016), and therefore, the intronless 35S:TERT construct may produce a suboptimal amount of TERT. Alternatively, the $35 \mathrm{~S}$ promoter may not be efficiently active in tissues where telomerase is most needed, such as rapidly growing embryos (Sunilkumar, Mohr et al., 2002).

Although the hypomorphic TERT allele could not restore telomeres to wild type length, it was capable of maintaining telomeres at a shorter length set point. This is evidenced by the fact that 35S:TERT plants can survive for 18 generations, and once the new shorter telomere length set point is established around G10/T6, plants maintain that telomere length for multiple generations. We confirmed that telomere length maintenance requires the catalytic activity of TERT since plants bearing 
a catalytically dead reverse transcriptase could not survive beyond the normal lifespan of tert mutants (G8-G10). Thus, while the hypomorphic 35S:TERT allele is sufficient to indefinitely prolong plant lifespan, it can only sustain telomeres close to their minimal functional length. Additional support for this conclusion comes from the observation that in late generation 35S:TERT lines deficient in the POT1A processivity factor lethality is immediate. We conclude that critically short telomeres in these lines cannot be maintained by telomerase and the plants succumb to telomere dysfunction.

The telomeres of late generation 35S:TERT lines were maintained at a bimodal size distribution with arm-specific lengths corresponding to either 400 (average 421, arms $1 \mathrm{~L}, 1 \mathrm{R}, 3 \mathrm{~L}, 5 \mathrm{~L}$, and $5 R$ ) or 900 bp (910, arms $2 \mathrm{R}$ and $4 \mathrm{R})$. FISH analysis unexpectedly demonstrated the shorter telomeres were no more prone to fusion than longer telomeres, implying that, despite their longer length, the capping status of the $2 R$ and $4 R$ telomeres is similar to other arms. Interestingly, the $2 R$ and $4 R$ telomeres are also maintained at longer lengths in wild type plants expressing normal TERT. Hence, cis-regulatory elements on these arms may not only modulate capping status, but also telomere length. A unique feature of the $2 R$ and $4 R$ telomeres is the immediate proximity of abundantly expressed protein coding genes (Vrbsky, Akimcheva et al., 2010), indicating that transcription of canonical transcripts may influence the status of neighboring telomeres. We note that regulation of telomere length by cis elements has been reported in humans (Britt-Compton, Rowson et al., 2006).

Despite extensive analysis of chromosome end protection in yeast and mammals, the relationship between the length of a telomere and its ability to serve as a functional cap on the DNA terminus remains enigmatic. Previous efforts to define the minimal functional length of telomeres have relied on identification of the shortest telomeres in a population of cells undergoing telomere crisis (Capper et al., 2007, Heacock et al., 2004, Heacock, Idol et al., 2007, Xu \& Blackburn, 2007). A drawback to this approach is that cannot assess whether very short telomeres were captured as they were being processed by DNA repair machinery, or were the result of compromised checkpoint pathways. Other experiments have measured the amount of telomeric DNA retained at cloned chromosome fusion junctions, but in these cases it is impossible to determine telomere length prior to fusion (Heacock et al., 2004, Heacock et al., 2007). In contrast, in 35S:TERT lines all telomeres are maintained at a length sufficient to confer long-term survival, but insufficient for buffering any additional loss of telomeric repeats.

For human cells, previous estimates of the minimal functional telomere size are between 42 (Xu \& Blackburn, 2007) and $72 \mathrm{nt}$ (Capper et al., 2007), lengths which are still sufficient for binding at least one TRF1 or TRF2 molecule. These observations, along with the fact that $72 \mathrm{nt}$ of telomeric DNA bound by TRF2 could inhibit NHEJ in vitro (Bae \& Baumann, 2007), have led to the proposal that the minimal functional length is defined by the ability to bind TRF2. Similarly, yeast cells continue to grow and divide until telomeres reach less than $100 \mathrm{bp}$ (Forstemann et al., 2000). Our results argue that the minimal functional length in Arabidopsis is significantly longer at approximately $400 \mathrm{bp}$, consistent with previous analysis of telomere fusion junctions reporting a minimal functional length of $360 \mathrm{bp}$ (Heacock et al., 2004). While several telomere-specific binding components have been described for Arabidopsis (Schrumpfova, Vychodilova et al., 2014, Song, Leehy et al., 2008, Surovtseva, Churikov et al., 2009), the limited biochemical data available for these factors suggest the binding sites are similar to the yeast and mammalian telomere proteins, and hence much smaller than $400 \mathrm{bp}$. In contrast, 400 $b p$ is the approximate length required for circularization of double-stranded DNA. Experiments on naked DNA have suggested that DNA molecules with lengths of roughly 300-400 bp are optimal for circular ligation (Shore, Langowski et al., 1981). In addition, in vitro experiments with TRF2 revealed 
that telomeric tracts of $500 \mathrm{bp}$ are sufficient to form t-loops (Griffith et al., 1999, Stansel, de Lange et al., 2001). Although the length of looped DNA in a t-loop is proportional to total telomere length, the loops in the $500 \mathrm{bp}$ artificial constructs averaged $338 \mathrm{bp}$, very close to the minimal telomere length detected at multiple chromosome arms in this study. Therefore, we postulate that formation of a secondary structure such as a t-loop is required for proper capping of chromosome ends, and thus defines the minimal functional length for telomeres in Arabidopsis.

\section{Materials and Methods}

Plants and Growth Conditions

Plants were grown in soil under long day conditions with $16 \mathrm{hr}$ daylight at $21^{\circ} \mathrm{C}$. All mutants and genotyping conditions have been previously described: tert (Fitzgerald, Riha et al., 1999), ku70 (Riha et al., 2002), pot1a (Surovtseva et al., 2007).

\section{Constructs and plant transformation}

Full length TERT CDNA was obtained from reverse-transcription reactions from wild type RNA using the RevertAid First Strand CDNA Synthesis Kit and amplified with Phusion Hot Start II polymerase (Fermentas) using primers TERT cDNA 5' BamHI (5'- GGATCCAAGGAGGAGGAAGGTGTAATG-3') and TERT cDNA 3' Sacl (5'- GAGCTCAAATGTTACAAATCCATTTTG-3'). Full length cDNA products were subcloned into PCR2.1-TOPO (Invitrogen) and sequenced. The CDNA was then transferred to binary vectors pCBK05 (35S promoter) forming pCBJ05 or pCBM10 (Actin promoter) forming pCBJ06 using BamHI and Sacl sites. For the D680N mutation, pCBJ05 was digested with HindIII, releasing a small fragment containing the catalytic residue, and subcloned into pBluescript. Mutagenesis was performed using the QuikChange site directed mutagenesis kit (Stratagene) using manufacturer's instructions and the primers AtD860NF(5'-GAGATTTATTAATGACTACCTTTTTG-3') and AtD860NR (5'CAAAAAGGTAGTCATTAATAAATCTC-3') resulting in a change from GAT to AAT. The mutated fragment was re-inserted into $\mathrm{pCBJ05}$. Transformation of G4 tert mutants was performed by the floral dip method and transformed plants were selected for resistance to the herbicide Basta.

\section{TRAP assay}

TRAP assays were performed as previously described (Kannan, Nelson et al., 2008).

\section{Telomere length analysis}

DNA extraction and TRF analysis were performed as previously described (Watson \& Shippen, 2007). For both PETRA (Heacock et al., 2004) and STELA (Baird, Rowson et al., 2003) 20 ng of total genomic DNA were used. For PETRA, the PETRA-T primer (5'-CTCTAGACTGTGAGACTTGGACTACCCTAAACCCT$\left.3^{\prime}\right)$ was annealed to G-overhangs and extended in a $20 \mu \mathrm{L}$ reaction containing $1 x$ Phi29 buffer (Fermentas), $125 \mu \mathrm{M}$ dNTPs, $0.5 \mu \mathrm{M}$ PETRA-T, and $4 \mathrm{U}$ Phi29 at $30^{\circ} \mathrm{C}$ for $1 \mathrm{hr}$. Samples were heat inactivated at $65^{\circ} \mathrm{C}$ for 20 minutes, precipitated with ethanol, and resuspended in $20 \mu \mathrm{L}$ of water. For STELA, the primer Fok2 (5'-CTCTAGACTGTGAGACTTGGACTACAGGATGTAAACCC- $3^{\prime}$ ) was ligated to genomic DNA in $20 \mu \mathrm{L}$ reactions containing 1x T4 DNA ligase buffer, $0.5 \mu \mathrm{M}$ Fok2, and $1 \mu \mathrm{L}$ T4 DNA ligase (Fermentas) overnight at $16^{\circ} \mathrm{C}$. Samples were heat inactivated at $65^{\circ} \mathrm{C}$ for 20 min, precipitated with ethanol, and resuspended in $20 \mu \mathrm{L}$ of water. Following this initial processing, STELA and PETRA reactions were identical. $1 \mu \mathrm{L}$ of STELA or PETRA products were PCR amplified in $20 \mu \mathrm{L}$ reactions 
containing 1x Phusion GC buffer, $200 \mu \mathrm{M}$ dNTPs, $0.5 \mu \mathrm{M}$ PETRA-A (5'-CTCTAGACTGTGAGACTTGGACTA C-3'), $0.5 \mu \mathrm{M}$ of one subtelomere specific primer (1L: 5'-AGGACCATCCCATATCATTGAGAGA-3', 1R: 5'CTATTGCCA GAACCTTGATATTCAT-3'， 2R: 5'-CAACATGGCCCATTTAAGATTGAACGGC-3', 3L: 5'CATAATTCTCACAG CAGCACCGTAGA-3', 4R: 5'-TGGGTGATTGTCATGCTACATGGTA-3', 5L: 5'AGGTAGAGTGAACCTAACA CTTGG-3', 5R: 5'-CAGGACGTGTGAAACAGAAACTACA-3'), 0.4 U Phusion Hot Start II (Fermentas). PCR reactions were performed by incubating samples at $98^{\circ} \mathrm{C}$ for 3 min followed by $22-25 \mathrm{cycles}$ of $98^{\circ} \mathrm{C} 15 \mathrm{sec}, 60^{\circ} \mathrm{C} 30 \mathrm{sec}, 72^{\circ} \mathrm{C} 2 \mathrm{~min} 30 \mathrm{sec}$, followed by a final incubation at $72^{\circ} \mathrm{C}$ for $10 \mathrm{~min}$. Reactions were then separated on $1.2 \%$ agarose gels cast in $12 \times 14 \mathrm{~cm}$ trays at $100 \mathrm{~V}$ for 2 h $30 \mathrm{~min}$. DNA was transferred to nylon membranes by Southern blotting. Hybridization was performed as previously described (Heacock et al., 2004), membranes were exposed to Kodak phosphoscreens, visualized with a Pharos FX Plus (Biorad) and images were analyzed with Imagelab (Biorad).

Chromosome Preparation, Probe Labeling, BAC FISH and Image Processing

Whole inflorescences of Arabidopsis thaliana were fixed in freshly prepared ethanol:acetic acid fixative (3:1) overnight, transferred into $70 \%$ ethanol and stored at $-20^{\circ} \mathrm{C}$ until use. Selected inflorescences were rinsed in distilled water and citrate buffer ( $10 \mathrm{mM}$ sodium citrate, $\mathrm{pH} 4.8$ ), and digested by a $0.3 \%$ mix of pectolytic enzymes (cellulase, cytohelicase, pectolyase; all from Sigma) in citrate buffer for c. 3 hrs. Chromosome preparations were prepared from pistils and pretreated with RNase $(100 \mu \mathrm{g} / \mathrm{ml}$, AppliChem) and pepsin $(0.1 \mathrm{mg} / \mathrm{ml}$, Sigma-Aldrich) as previously described (Mandakova, Marhold et al., 2014). Arabidopsis thaliana BAC clones T25K16 (AC007323), F6F3 (AC023628), F22L4 (AC061957), T1N6 (AC009273) and F22N6 (B98711) were used for in situ identification of chromosome arm 1L, F19K16 (AC011717), F18B13 (AC009322), F516 (AC018848), T21F11 (AC018849) and F23A5 (AC011713) for 1R, F13A10 (AC006418), T3A4 (AC005819), F19D11 (AC005310), F14M4 (AC004411) and T8113 (AC002337) for 2R, and F19H22 (AL035679), T22F8 (AL050351), F23K16 (AL078620), T23P19 (B98327) and T5J17 (AL035708) for 4R. DNA probes were labeled with biotin-dUTP and digoxigenin-dUTP by nick translation as previously described (Mandakova, Joly et al., 2010). Differentially labeled BACs corresponding to $1 \mathrm{~L}+4 \mathrm{R}$ and $1 \mathrm{R}+2 \mathrm{R}$ chromosome arms were pooled, precipitated, and resuspended in $20 \mu$ l of hybridization mixture ( $50 \%$ formamide and $10 \%$ dextran sulfate in $2 \times S S C$ ) per slide. Probes and chromosomes were denatured together on a hot plate at $80^{\circ} \mathrm{C}$ for $2 \mathrm{~min}$ and incubated in a moist chamber at $37^{\circ} \mathrm{C}$ overnight. Post hybridization washing was performed in $20 \%$ formamide in $2 \times$ SSC at $42^{\circ} \mathrm{C}$. Fluorescent detection was as follows: biotin-dUTP was detected by avidin-Texas Red (Vector Laboratories) and amplified by goat anti-avidin-biotin (Vector Laboratories) and avidin-Texas Red; digoxigenin-dUTP was detected by mouse antidigoxigenin (Jackson ImmunoResearch) and goat antimouse Alexa Fluor 488 (Molecular Probes). Chromosomes were counterstained with DAPI (4',6diamidino-2-phenylindole; $2 \mu \mathrm{g} / \mathrm{ml}$ ) in Vectashield (Vector Laboratories). Fluorescence signals were analyzed with an Olympus BX-61 epifluorescence microscope and CoolCube CCD camera (MetaSystems). Images were acquired separately for the two fluorochromes using appropriate excitation and emission filters (AHF Analysentechnik). The two monochromatic images were pseudo colored and merged using Adobe Photoshop CS2 software (Adobe Systems).

\section{Acknowledgements}

The work was supported from the European Regional Development Fund-Project „REMAP“ (No. CZ.02.1.01/0.0/0.0/15_003/0000479), by the Czech Science Foundation (18-20134S), by EMBO 
Installation Grant (1304130933), by the Austrian Academy of Sciences and by the National Institutes of Health (GM R01-GM065383).

\section{References}

Bae NS, Baumann P (2007) A RAP1/TRF2 complex inhibits nonhomologous end-joining at human telomeric DNA ends. Mol Cell 26: 323-34

Baird DM, Rowson J, Wynford-Thomas D, Kipling D (2003) Extensive allelic variation and ultrashort telomeres in senescent human cells. Nat Genet 33: 203-207

Blasco MA (2002) Telomerase beyond telomeres. Nat Rev Cancer 2: 627-33.

Blasco MA, Lee HW, Hande MP, Samper E, Lansdorp PM, DePinho RA, Greider CW (1997) Telomere shortening and tumor formation by mouse cells lacking telomerase RNA. Cell 91: 25-34.

Britt-Compton B, Rowson J, Locke M, Mackenzie I, Kipling D, Baird DM (2006) Structural stability and chromosome-specific telomere length is governed by cis-acting determinants in humans. Hum Mol Genet 15: 725-33

Capper R, Britt-Compton B, Tankimanova M, Rowson J, Letsolo B, Man S, Haughton M, Baird DM (2007) The nature of telomere fusion and a definition of the critical telomere length in human cells. Genes Dev 21: 2495-508

Cesare AJ, Quinney N, Willcox S, Subramanian D, Griffith JD (2003) Telomere looping in P. sativum (common garden pea). Plant J 36: 271-9

de Lange T (2010) How shelterin solves the telomere end-protection problem. Cold Spring Harb Symp Quant Biol 75: 167-77

Doksani Y, Wu JY, de Lange T, Zhuang X (2013) Super-Resolution Fluorescence Imaging of Telomeres Reveals TRF2-Dependent T-loop Formation. Cell 155: 345-356

Fajkus J, Kovarik A, Kralovics R, Bezdek M (1995) Organization of telomeric and subtelomeric chromatin in the higher plant Nicotiana tabacum. Mol Gen Genet 247: 633-8.

Fitzgerald MS, Riha K, Gao F, Ren S, McKnight TD, Shippen DE (1999) Disruption of the telomerase catalytic subunit gene from Arabidopsis inactivates telomerase and leads to a slow loss of telomeric DNA. Proc Natl Acad Sci U S A 96: 14813-8.

Forstemann K, Hoss M, Lingner J (2000) Telomerase-dependent repeat divergence at the 3' ends of yeast telomeres. Nucleic Acids Res 28: 2690-4

Fouquerel E, Parikh D, Opresko P (2016) DNA damage processing at telomeres: The ends justify the means. DNA Repair (Amst) 44: 159-168

Fulcher N, Derboven E, Valuchova S, Riha K (2014) If the cap fits, wear it: an overview of telomeric structures over evolution. Cell Mol Life Sci 71: 847-65

Fulcher N, Teubenbacher A, Kerdaffrec E, Farlow A, Nordborg M, Riha K (2015) Genetic architecture of natural variation of telomere length in Arabidopsis thaliana. Genetics 199: 625-35

Greetham M, Skordalakes E, Lydall D, Connolly BA (2015) The Telomere Binding Protein Cdc13 and the Single-Stranded DNA Binding Protein RPA Protect Telomeric DNA from Resection by Exonucleases. J Mol Biol 427: 3023-30

Greider CW, Blackburn EH (1989) A telomeric sequence in the RNA of Tetrahymena telomerase required for telomere repeat synthesis. Nature 337: 331-7

Griffith JD, Comeau L, Rosenfield S, Stansel RM, Bianchi A, Moss H, de Lange T (1999) Mammalian telomeres end in a large duplex loop. Cell 97: 503-14.

Heacock M, Spangler E, Riha K, Puizina J, Shippen DE (2004) Molecular analysis of telomere fusions in Arabidopsis: multiple pathways for chromosome end-joining. Embo J 23: 2304-13 
Heacock ML, Idol RA, Friesner JD, Britt AB, Shippen DE (2007) Telomere dynamics and fusion of critically shortened telomeres in plants lacking DNA ligase IV. Nucleic Acids Res 35: 6490-500

Kannan K, Nelson AD, Shippen DE (2008) Dyskerin is a component of the Arabidopsis telomerase RNP required for telomere maintenance. Mol Cell Biol 28: 2332-41

Kazda A, Zellinger B, Rossler M, Derboven E, Kusenda B, Riha K (2012) Chromosome end protection by blunt-ended telomeres. Genes Dev 26: 1703-13

Laxa M (2016) Intron-Mediated Enhancement: A Tool for Heterologous Gene Expression in Plants? Front Plant Sci 7: 1977

Li Y, Tergaonkar V (2014) Noncanonical functions of telomerase: implications in telomerase-targeted cancer therapies. Cancer Res 74: 1639-44

Liti G, Haricharan S, Cubillos FA, Tierney AL, Sharp S, Bertuch AA, Parts L, Bailes E, Louis EJ (2009) Segregating YKU80 and TLC1 alleles underlying natural variation in telomere properties in wild yeast. PLoS Genet 5: e1000659

Luke-Glaser S, Poschke H, Luke B (2012) Getting in (and out of) the loop: regulating higher order telomere structures. Front Oncol 2: 180

Lundblad V, Szostak JW (1989) A mutant with a defect in telomere elongation leads to senescence in yeast. Cell 57: 633-43

Mandakova T, Joly S, Krzywinski M, Mummenhoff K, Lysak MA (2010) Fast diploidization in close mesopolyploid relatives of Arabidopsis. Plant Cell 22: 2277-90

Mandakova T, Marhold K, Lysak MA (2014) The widespread crucifer species Cardamine flexuosa is an allotetraploid with a conserved subgenomic structure. New Phytol 201: 982-92

Meier B, Clejan I, Liu Y, Lowden M, Gartner A, Hodgkin J, Ahmed S (2006) trt-1 is the Caenorhabditis elegans catalytic subunit of telomerase. PLoS Genet 2: e18

Munoz-Jordan JL, Cross GA, de Lange T, Griffith JD (2001) t-loops at trypanosome telomeres. Embo J 20: 579-88.

Nakamura TM, Morin GB, Chapman KB, Weinrich SL, Andrews WH, Lingner J, Harley CB, Cech TR (1997) Telomerase catalytic subunit homologs from fission yeast and human. Science 277: 9559.

Nakayama J, Tahara H, Tahara E, Saito M, Ito K, Nakamura H, Nakanishi T, Tahara E, Ide T, Ishikawa F (1998) Telomerase activation by hTRT in human normal fibroblasts and hepatocellular carcinomas. Nat Genet 18: 65-8

Raices M, Verdun RE, Compton SA, Haggblom Cl, Griffith JD, Dillin A, Karlseder J (2008) C. elegans telomeres contain $\mathrm{G}$-strand and $\mathrm{C}$-strand overhangs that are bound by distinct proteins. Cell 132: $745-57$

Renfrew KB, Song X, Lee JR, Arora A, Shippen DE (2014) POT1a and components of CST engage telomerase and regulate its activity in Arabidopsis. PLoS Genet 10: e1004738

Riha K, McKnight TD, Griffing LR, Shippen DE (2001) Living with genome instability: plant responses to telomere dysfunction. Science 291: 1797-800.

Riha K, Shippen DE (2003) Ku is required for telomeric C-rich strand maintenance but not for end-toend chromosome fusions in Arabidopsis. Proc Natl Acad Sci U S A 100: 611-5.

Riha K, Watson JM, Parkey J, Shippen DE (2002) Telomere length deregulation and enhanced sensitivity to genotoxic stress in Arabidopsis mutants deficient in Ku70. Embo J 21: 2819-26

Schrumpfova PP, Vychodilova I, Dvorackova M, Majerska J, Dokladal L, Schorova S, Fajkus J (2014) Telomere repeat binding proteins are functional components of Arabidopsis telomeres and interact with telomerase. Plant J 77: 770-81 
Sfeir A, de Lange T (2012) Removal of shelterin reveals the telomere end-protection problem. Science 336: 593-7

Shakirov EV, Shippen DE (2004) Length regulation and dynamics of individual telomere tracts in wildtype Arabidopsis. Plant Cell 16: 1959-67

Shore D, Langowski J, Baldwin RL (1981) DNA flexibility studied by covalent closure of short fragments into circles. Proc Natl Acad Sci U S A 78: 4833-7

Song X, Leehy K, Warrington RT, Lamb JC, Surovtseva YV, Shippen DE (2008) STN1 protects chromosome ends in Arabidopsis thaliana. Proc Natl Acad Sci U S A 105: 19815-20

Soudet J, Jolivet P, Teixeira MT (2014) Elucidation of the DNA end-replication problem in Saccharomyces cerevisiae. Mol Cell 53: 954-64

Stansel RM, de Lange T, Griffith JD (2001) T-loop assembly in vitro involves binding of TRF2 near the 3 ' telomeric overhang. EMBO J 20: 5532-40

Stewart JA, Chaiken MF, Wang F, Price CM (2012) Maintaining the end: roles of telomere proteins in end-protection, telomere replication and length regulation. Mutat Res 730: 12-9

Sunilkumar G, Mohr L, Lopata-Finch E, Emani C, Rathore KS (2002) Developmental and tissue-specific expression of CaMV 35 S promoter in cotton as revealed by GFP. Plant Mol Biol 50: 463-74

Surovtseva YV, Churikov D, Boltz KA, Song X, Lamb JC, Warrington R, Leehy K, Heacock M, Price CM, Shippen DE (2009) Conserved telomere maintenance component 1 interacts with STN1 and maintains chromosome ends in higher eukaryotes. Mol Cell 36: 207-18

Surovtseva YV, Shakirov EV, Vespa L, Osbun N, Song X, Shippen DE (2007) Arabidopsis POT1 associates with the telomerase RNP and is required for telomere maintenance. Embo J 26: 365361

Teixeira MT, Arneric M, Sperisen P, Lingner J (2004) Telomere length homeostasis is achieved via a switch between telomerase- extendible and -nonextendible states. Cell 117: 323-35

Tomaska L, Makhov AM, Griffith JD, Nosek J (2002) t-Loops in yeast mitochondria. Mitochondrion 1: 455-9

Tomaska L, Willcox S, Slezakova J, Nosek J, Griffith JD (2004) Taz1 Binding to a Fission Yeast Model Telomere: FORMATION OF TELOMERIC LOOPS AND HIGHER ORDER STRUCTURES. J Biol Chem 279: 50764-72

Tomita K (2018) How long does telomerase extend telomeres? Regulation of telomerase release and telomere length homeostasis. Curr Genet 64: 1177-1181

Vrbsky J, Akimcheva S, Watson JM, Turner TL, Daxinger L, Vyskot B, Aufsatz W, Riha K (2010) siRNAmediated methylation of Arabidopsis telomeres. PLoS Genet 6: e1000986

Watson JM, Shippen DE (2007) Telomere rapid deletion regulates telomere length in Arabidopsis thaliana. Mol Cell Biol 27: 1706-15

Xu L, Blackburn EH (2007) Human cancer cells harbor T-stumps, a distinct class of extremely short telomeres. Mol Cell 28: 315-27 


\section{Figure Legends}

Fig 1. Phenotypic analysis of the 35S:TERT allele. (A) TRAP assay of wild type seedlings and seedlings transformed with either 35S:TERT or ACT2:TERT constructs. Both constructs show reduced telomerase activity relative to wild type. (B) TRF analysis of successive generations of 35S:TERT or ACT2:TERT plants. Wild type and tert mutants are shown as controls; the asterisk marks interstitial telomeric DNA. Rescue constructs were transformed into G4 tert mutants. The " $\mathrm{T}$ " designation indicates the number of generations post transformation (Fig S1). (C) Phenotypic analysis of successive generations of plants containing the 35S:TERT construct. Two independent lines were analyzed (line 2 and 7) with G6/T2, G7/T3 etc. The total number of plants analyzed in each population is indicated below the bars. (D) Percentage of anaphases containing chromatin bridges over successive generations of plants containing the 35S:TERT construct. Total number of anaphases examined is indicated. Data represents pools from both lines.

Fig 2. Genetic analysis of the 35S:TERT allele. (A) Phenotypic analysis of successive generations of 35S:TERT(D680N) transformants. In the absence of telomerase catalytic activity, two independent transformant lines (5 and 17) show rapid loss of viability. (B) TRF analysis of 35S:TERT containing siblings segregating for $k u 70$ (see Fig. S4 for details of the cross). Loss of KU7O leads to immediate lengthening of telomeres. Asterisks mark interstitial telomeric DNA. (C) Phenotypic analysis of 35S:TERT plants segregating for POT1A. See Fig. 55 for details for the cross. Loss of POT1A increases severity of the phenotypes in 35S:TERT background.

Fig 3. Analysis of individual telomeres in 35S:TERT plants. (A) Measurement of individual telomere lengths over successive generations in lines 2 and 7 by PETRA. Measurements are the average of four plants, with the exception of G6/T2 in line where only one plant was available, and G12/T8 and G14/T10 where five plants were measured. (B) Comparison of telomere lengths in G14/T10 plants. Data is the same as in $(A)$ but plotted as a bar graph for clarity. Error bars represent standard deviation for all data points in $(A)$. (C) Representative figures of FISH analysis. Anaphase bridges showing telomere fusions of $2 R$ and $4 R$ are shown.

Fig 4. Single telomere analysis by STELA in wild type plants. $(A)$ Telomere length as measured by STELA. Data represents the average of 32 plants. Error bars represent standard error of the mean. $(B)$ Average telomere rank. As the average telomere length between plants varied, we ranked telomeres within each plant, telomeres were ranked from $1=$ shortest to $7=$ longest and the ranks were averaged. Error bars represent standard error of the mean. 
Table 1. Dual label FISH analysis of anaphase bridges in Type II G14 /T10 mutants with probes recognizing either chromosome arms $4 R$ and $5 \mathrm{~L}$, or $2 R$ and $1 R$. No significant difference was observed between telomeres $4 \mathrm{R}$ (short) and $1 \mathrm{~L}$ (long), and between $2 \mathrm{R}$ (long) and $1 \mathrm{R}$ (short).

\begin{tabular}{ccccc} 
& $\begin{array}{c}\text { Anaphases with } \\
\text { bridges }\end{array}$ & Bridges with signal & Percentage & $\begin{array}{c}\text { Fisher's exact } \\
\text { test }\end{array}$ \\
$4 \mathrm{R}$ & 55 & 8 & 14,50 & 0,3879 \\
$1 \mathrm{~L}$ & 55 & 6 & 10,9 & \\
\hline $2 \mathrm{R}$ & 52 & 20 & 38,5 & 0,34534 \\
$1 \mathrm{R}$ & 52 & 23 & 44,2 &
\end{tabular}




\section{Figures}

Fig 1. Phenotypic analysis of the 35S:TERT allele. (A) TRAP assay of wild type seedlings and seedlings transformed with either 35S:TERT or ACT2:TERT constructs. Both constructs show reduced telomerase activity relative to wild type. (B) TRF analysis of successive generations of 35S:TERT or ACT2:TERT plants. Wild type and tert mutants are shown as controls; the asterisk marks interstitial telomeric DNA. Rescue constructs were transformed into G4 tert mutants. The " $T$ " designation indicates the number of generations post transformation (Fig S1). (C) Phenotypic analysis of successive generations of plants containing the 35S:TERT construct. Two independent lines were analyzed (line 2 and 7) with G6/T2, G7/T3 etc. The total number of plants analyzed in each population is indicated below the bars. (D) Percentage of anaphases containing chromatin bridges over successive generations of plants containing the 35S:TERT construct. Total number of anaphases examined is indicated. Data represents pools from both lines.

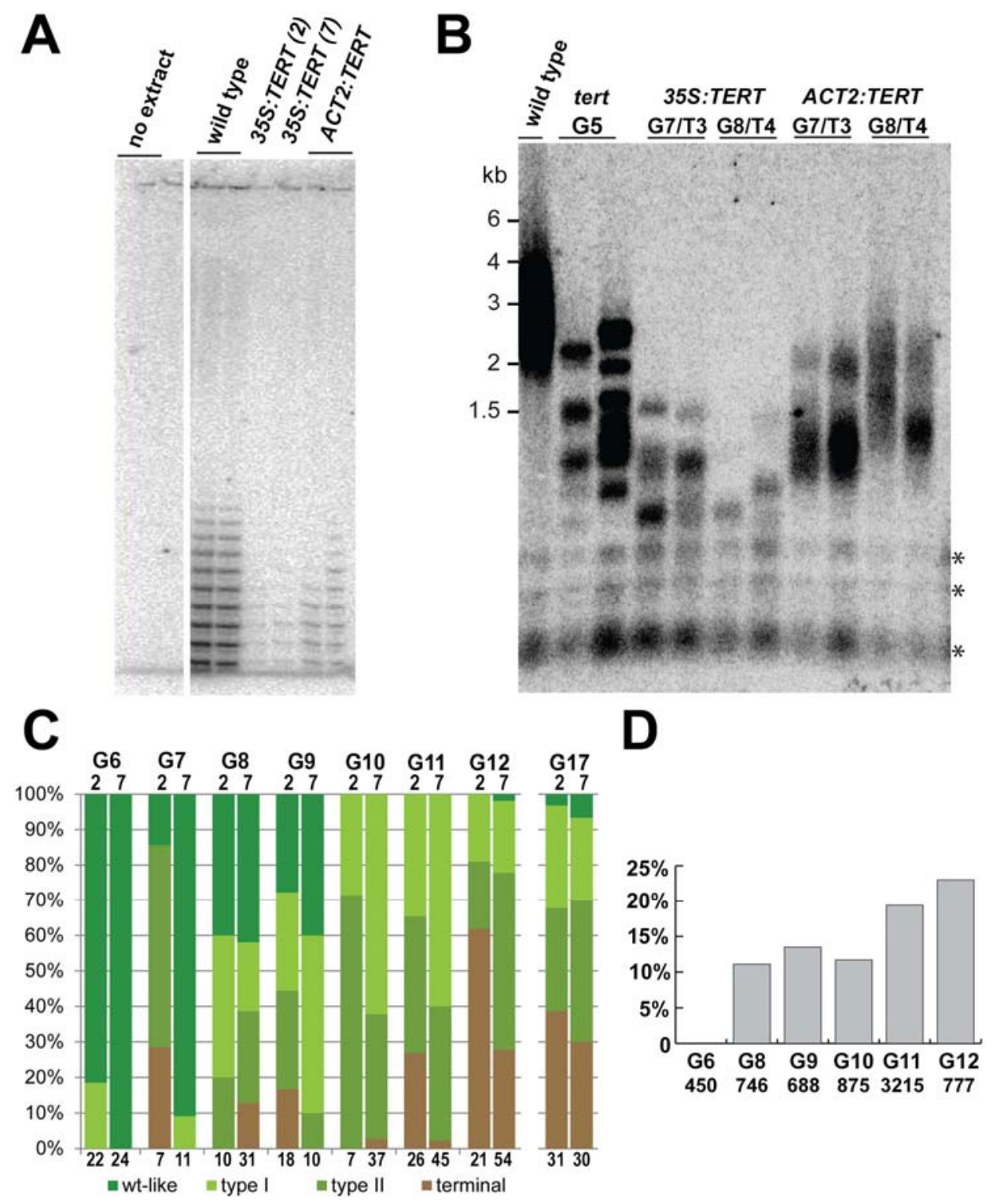


Fig 2. Genetic analysis of the 35S:TERT allele. (A) Phenotypic analysis of successive generations of 35S:TERT(D680N) transformants. In the absence of telomerase catalytic activity, two independent transformant lines (5 and 17) show rapid loss of viability. (B) TRF analysis of 35S:TERT containing siblings segregating for $k u 70$ (see Fig. S4 for details of the cross). Loss of KU7O leads to immediate lengthening of telomeres. Asterisks mark interstitial telomeric DNA. (C) Phenotypic analysis of 35S:TERT plants segregating for POT1A. See Fig. S5 for details for the cross. Loss of POT1A increases severity of the phenotypes in 35S:TERT background.
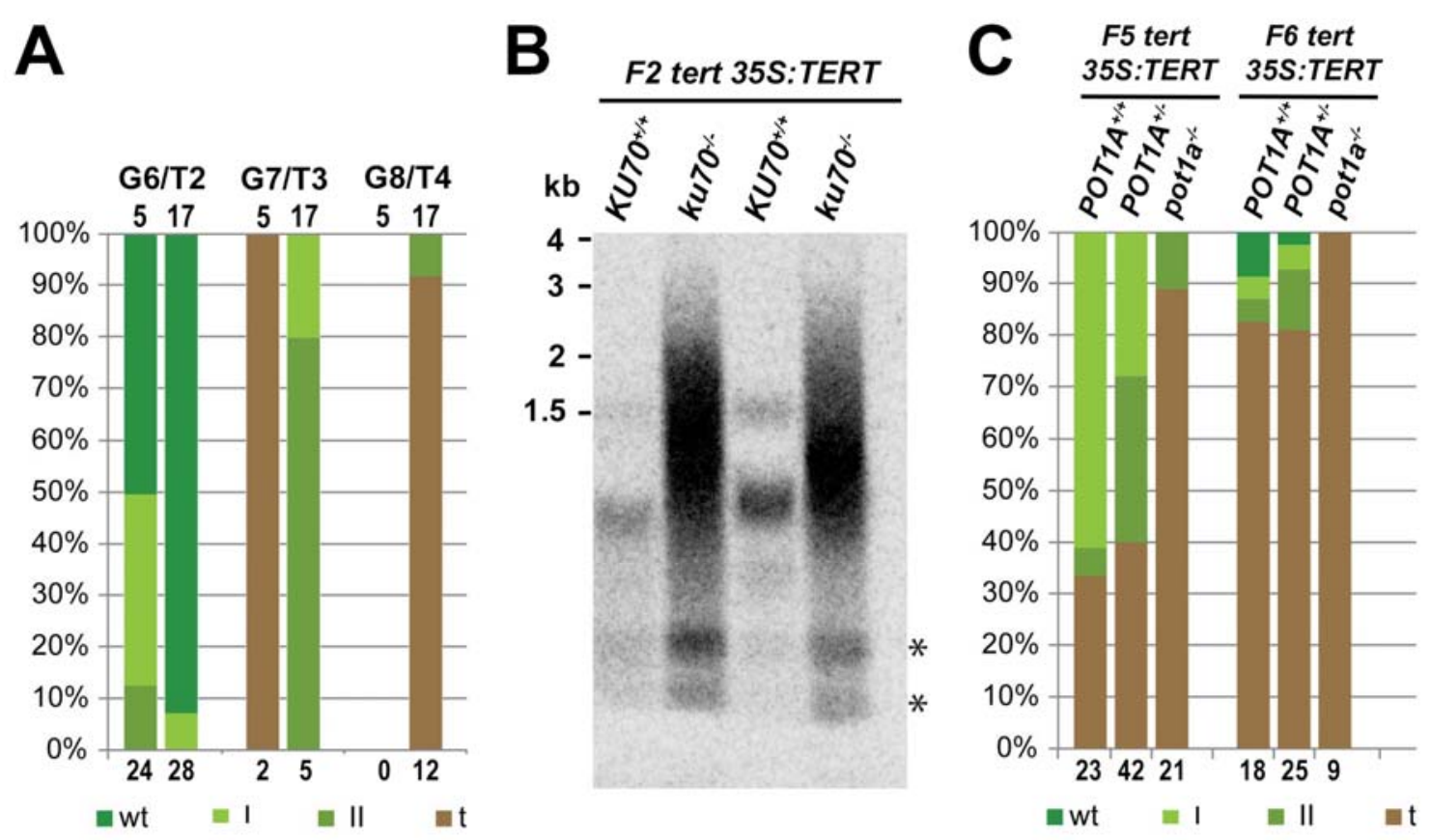
Fig 3. Analysis of individual telomeres in 35S:TERT plants. (A) Measurement of individual telomere lengths over successive generations in lines 2 and 7 by PETRA. Measurements are the average of four plants, with the exception of G6/T2 in line where only one plant was available, and G12/T8 and G14/T10 where five plants were measured. (B) Comparison of telomere lengths in G14/T10 plants. Data is the same as in $(A)$ but plotted as a bar graph for clarity. Error bars represent standard deviation for all data points in $(A)$. (C) Representative figures of FISH analysis. Anaphase bridges showing telomere fusions of $2 \mathrm{R}$ and $4 \mathrm{R}$ are shown.
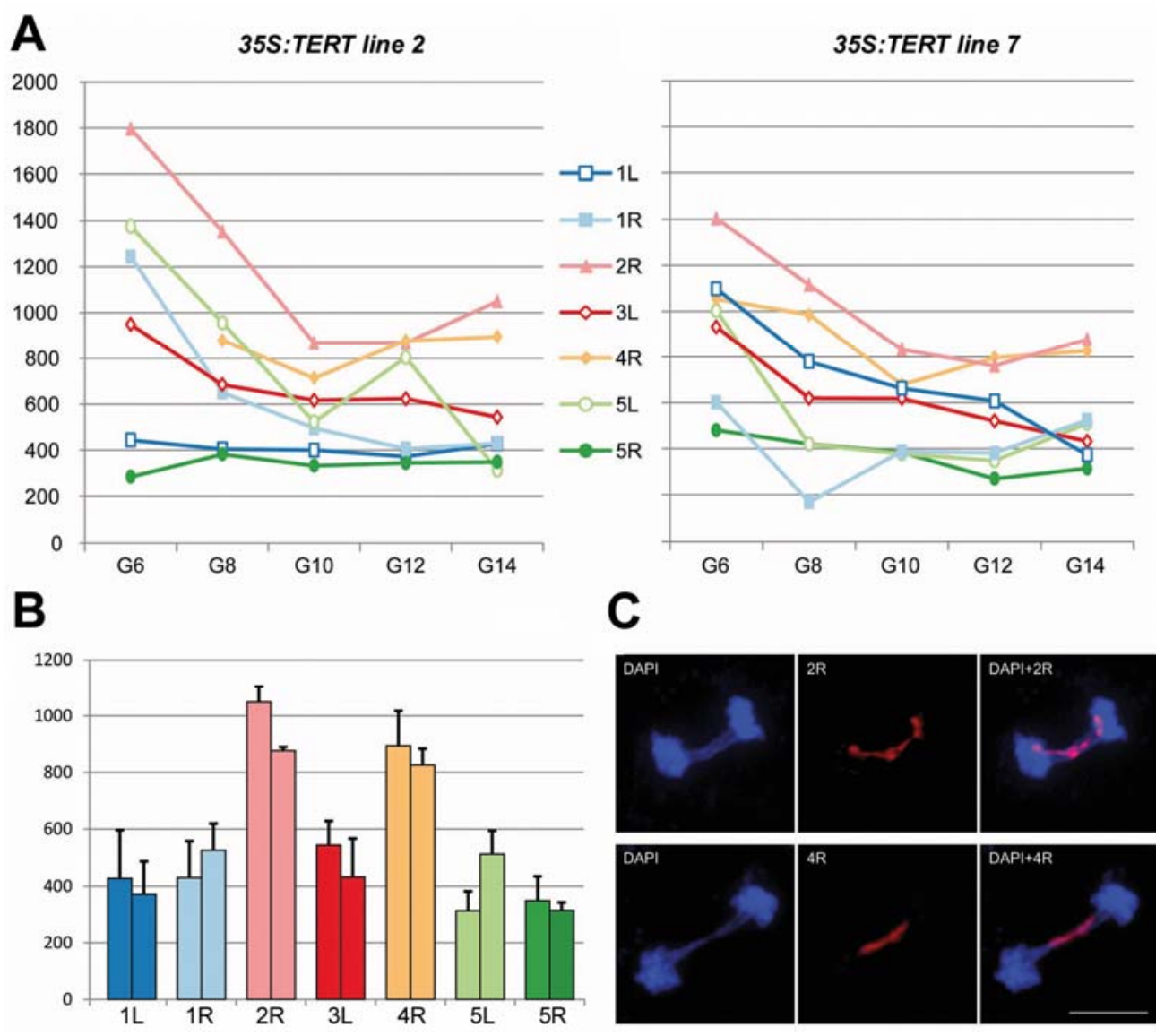
Fig 4. Single telomere analysis by STELA in wild type plants. $(A)$ Telomere length as measured by STELA. Data represents the average of 32 plants. Error bars represent standard error of the mean. (B) Average telomere rank. As the average telomere length between plants varied, we ranked telomeres within each plant, telomeres were ranked from $1=$ shortest to $7=$ longest and the ranks were averaged. Error bars represent standard error of the mean.
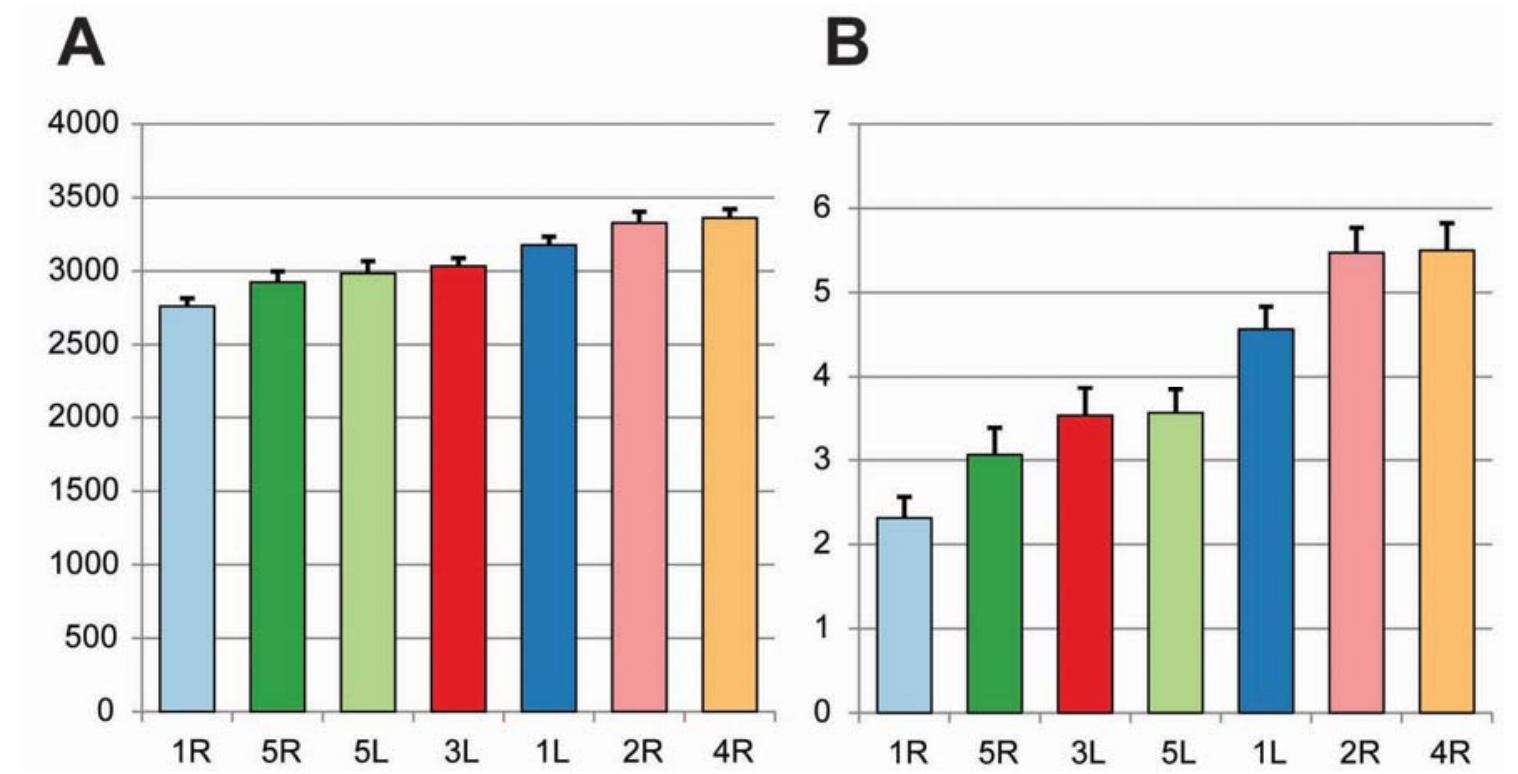